Vos, H.M.M., Bor, H.H., Rangelrooij-Minkels, M.J.A., Schellevis, F.G., Lagro-Janssen, A.L.M. Multimorbidity in older women: the negative impact of specific combinations of chronic

\begin{tabular}{l|l}
$\begin{array}{l}\text { Postprint } \\
\text { Version }\end{array}$ & 1.0 \\
\hline Journal website & $\underline{\text { http://informahealthcare.com/doi/abs/10.3109/13814788.2012.755511 }}$ \\
\hline Pubmed link & $\underline{\text { http://www.ncbi.nlm.nih.gov/pubmed/23336329 }}$ \\
DOI & $10.3109 / 13814788.2012 .755511$
\end{tabular}

This is a NIVEL certified Post Print, more info at http://www.nivel.eu

\title{
Multimorbidity in older women: The negative impact of Specific combinations of chronic conditions on self-rated health
}

\author{
HedWig M. M. $\operatorname{Vos}^{1}{ }^{\text {, HANS H. BOR }}{ }^{1}$, MARJOLEIN J.A. VAN RANGELROOIJ-MinKELS ${ }^{1}$, \\ FRAN Ç OIS G. SCHELLEVIS ${ }^{2,3}$ \& ANTOINE L. M. LAGRO-JANSSEN ${ }^{1}$ \\ ${ }^{1}$ Gender and Women's Health, Radboud University Nijmegen Medical Centre, Department \\ of Primary and Community Care, The Netherlands, \\ ${ }^{2}$ NIVEL (Netherlands Institute for Health Services Research), Utrecht, The Netherlands, and \\ ${ }^{3}$ Department of General Practice/EMGO Institute for Health and Care Research, VU \\ University Medical Centre, Amsterdam, The Netherlands
}

\begin{abstract}
Background: Chronic diseases are considered major threats to self-rated health (SRH). In many elderly people multimorbidity is present, in elderly women more than in elderly men. This study aims at establishing the impact of multimorbidity and specifi c disease combinations on SRH in elderly women . Objectives: To study the relationship between the number of chronic diseases and SRH and explore possible eff ects of combinations of chronic conditions on SRH in elderly women .

Methods: Health interview data used for this study originated from the second Dutch National Survey of General Practice, a study with a response rate of 64.5\%. From the 12699 respondents, 315 were females between 70 and 74 years old .

Results: Of the women, $87 \%$ reported one or more chronic condition. Women without any chronic condition rated their health signifi - cantly better than those with one or more chronic conditions. Either severe back pain or severe headache was included in return the most prevalent combinations of two chronic conditions with a signifi cantly higher negative impact on SRH than expected . Conclusion: All combinations including severe headache and some combinations including severe back pain and another chronic condition had a signifi cantly more negative impact on SRH than expected in women aged 70 74 years. General practitioners should be alert on severe headache and severe
\end{abstract}


Vos, H.M.M., Bor, H.H., Rangelrooij-Minkels, M.J.A., Schellevis, F.G., Lagro-Janssen, A.L.M. Multimorbidity in older women: the negative impact of specific combinations of chronic conditions on self-rated health. European Journal of General Practice: 2013, 19(2), 117-122

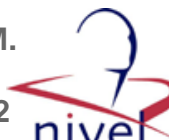

back pain in elderly women to improve proactive the quality of care and thus add to the quality of later years of life .

\section{INTRODUCTION}

A substantial proportion of the elderly suff ers from more than one chronic disease as there is a relation between increasing age and the incidence of multimorbidity (1) . Women suff er from more chronic conditions in old age than men (2). Higher prevalence rates of non-fatal disabling conditions contribute substantially more to disability and poor self-rated health (SRH) among aging women compared to aging men (2). Not only do women have more disabilities than men, they also have a longer lifespan characterized by a poor SRH $(3,4)$.

$\mathrm{SRH}$ is widely recognized as a comprehensive indicator of health $(5,6)$, and is adversely aff ected by multimorbidity (7). Some chronic conditions are more strongly associated with poor SRH than others, and some combinations of chronic conditions appear to have a more negative impact on SRH than expected (7). Gijsen et al., found that comorbid mental disorders were associated with poor SRH (8) . Other combinations of diseases known to aff ect inversely SRH are diabetes, cardiovascular disease and/or chronic respiratory disease $(7,9,10)$.

In general practice multimorbidity represents the rule rather than the exception among elderly patients (11). In the Netherlands, the general practitioner (GP) has a central position in health care as a gatekeeper to secondary care. General practice is, therefore, an important entrance to the health care system. To off er proactively guidance and treatment, and to improve the quality of care of patients with multimorbidity, GPs need to be aware and must have knowledge of combinations of conditions that negatively aff ect SRH .

Having a combination of two specifi c chronic conditions may have more impact than would be expected from having any combination of two or more chronic conditions and demonstrating the eff ect of multimorbidity is of great importance. The aim of this study was, therefore, to determine whether a signifi cantly higher impact on SRH of specifi c combinations of chronic conditions was present among most prevalent combinations of chronic conditions in women aged $70-74$ years in general practice to identify target groups for proactive action and alertness. We also studied the relationship between the number of chronic conditions and SRH . We selected women aged $70-74$ years for several reasons. From an anticipating point of view, at this age it is eff ectively possible to add to the quality of later years of life. Moreover, an older group would contain fewer respondents because of cognitive disability. Lastly, this group is, although mostly retired from work, considered being still active as volunteer, informal caregiver for their partner or baby sitter for their grandchildren and thus contributes largely to our social capital .

\section{METHODS}

The data used in this study originate from the Second Dutch National Survey of General Practice (DNSGP-2) of the Netherlands Institute for Health Services Research (NIVEL), which has been carried out in cooperation with the National Information Network of General Practice (NIN-GP) (12). The DNSGP-2 was carried out with the aim of providing information for researchers and policy makers about the role of general practice in the Dutch health care system. Data was collected 
Vos, H.M.M., Bor, H.H., Rangelrooij-Minkels, M.J.A., Schellevis, F.G., Lagro-Janssen, A.L.M. Multimorbidity in older women: the negative impact of specific combinations of chronic conditions on self-rated health. European Journal of General Practice: 2013, 19(2), 117-122

between April 2000 and January 2002. The study was carried out in 104 general practices in the Netherlands, comprising 195 GPs (in total 165 GP full-time equivalents). The listed patients in these practices ( $n_{-} 385461$ ) form a representative sample of the Dutch population. An all-age random sample of approximately five per cent of the Dutchspeaking listed patients was asked to participate in a health interview survey ( $n_{-} 19$ 685); 12699 responded (64.5\%). The 90 min computer assisted interviews were carried out at the homes of the respondents. To avoid seasonal patterns, health interviews were randomly distributed over the year. From the 12699 respondents, 315 were females between 70 and 74 years old .

\section{Self-rated health}

SRH was operationalized as the score on the general perceptions scale of the ShortForm 36. The question asked was: In general, would you say your health is: excellent, very good, good, fair or poor (13). The five categories were dichotomized into high SRH (excellent, very good and good health) versus low SRH (fair and poor health). Previously, a Dutch version of the SF-36 was validated (14) .

\section{Chronic conditions}

Participants in the health interview were asked whether they suffered from one or more chronic conditions from a fixed list in the 12 months prior to the interview. The chronic conditions are self-reported. The list of conditions was developed under the auspices of Statistics Netherlands and has been regularly applied in health surveys in the Netherlands in the past decades (12) .

\section{Multimorbidity}

To study the effects of multimorbidity on SRH, we selected the top five of chronic conditions and the most prevalent combinations of two chronic diseases containing at least one of these five most prevalent conditions .

Other combinations of chronic conditions not being part of the top five most prevalent combinations, but present in 20 or more women were also studied .

\section{Impact on self-rated health}

To determine the impact of a combination of two chronic conditions on SRH, we compared the SRH of women with a combination of at least two specific chronic conditions of the most prevalent combinations to the SRH of all women with any combination of two or more chronic conditions. For this, we calculated a SRH ratio. The numerator of the ratio is the proportion of the women suffering from two specific chronic conditions and reporting a high SRH. The denominator is the proportion of women suffering from any combination of two or more chronic conditions and reporting a high SRH. If the ratio is statistically significantly lower than 1.0, this indicates a negative effect of the combination of two specific chronic conditions on SRH. We considered a P value of less than 0.05 as statistically significant .

\section{Analyses}

Statistical analysis was performed with SPSS statistical software for Windows. Descriptive statistics (percentages) were calculated to describe the study population . Stratified cross table analysis was performed to test the relation between SRH and multimorbidity. Chi-square (goodness of fi t) testing was used to test for statistical significance. To produce the $95 \%$ confi dence intervals around the estimates of the 
Vos, H.M.M., Bor, H.H., Rangelrooij-Minkels, M.J.A., Schellevis, F.G., Lagro-Janssen, A.L.M. Multimorbidity in older women: the negative impact of specific combinations of chronic conditions on self-rated health. European Journal of General Practice: 2013, 19(2), 117-122

SRH-ratio, bootstrap sampling (1000 iterations) and the 95\% BCa-intervals were used. This analysis was conducted in R version 2.15.1 .

\section{RESULTS}

Data of 315 women aged 70 to 74 years were included .

The respondents were equally divided among these fi ve years of age. The mean age was 71.9 years (Table 1$)$.

Table 2 shows the chronic conditions reported by the participants during the interview. The fi ve most common chronic conditions were osteoarthritis of hip or knee, anxiety, hypertension, depression and urinary incontinence .

Almost nine out of ten women (87\%) reported one or more chronic conditions. Almost three quarters of the respondents rated her health as high. Twenty eight per cent rated her health as low. Respondents without any chronic condition signifi cantly more often rated their health as high as those with one or more chronic conditions ( $\mathrm{P}_{-}$0.024). When the number of chronic conditions increased, more respondents rated their health as low $\left(\mathrm{P}_{-}{ }_{0.001)}\right.$ (Figure 1$)$.

To determine the SRH-ratio the expected proportion of women with a good SRH and two or more chronic conditions was calculated: 122 of the total of 202 women with two or more chronic conditions rated their health as good. The expected proportion was, therefore, $60.4 \%$.

Several of the combinations of two chronic conditions that were selected revealed a statistically signifi cant negative impact on the SRH. Signifi cantly negative eff ects on SRH were seen in the following combinations of chronic conditions: severe back pain and osteoarthritis of hip or knee, SRH-ratio 0.65 ( $\mathrm{P}_{-}$0.022), severe back pain and depression, SRH-ratio 0.50 ( $\mathrm{P}_{-}$0.005), migraine/severe headache and urinary incontinence, SRH-ratio 0.35 ( $\mathrm{P}_{-}$0.003), and migraine/severe headache and anxiety, SRH-ratio 0.58 ( $\mathrm{P}_{-}$0.020). The combinations of severe back pain and hypertension, SRH-ratio 0.62 ( $\left.\mathrm{P}_{-} 0.061\right)$ and severe back pain and a severe condition of the neck/ shoulder, SRH-ratio 0.68 ( $\mathrm{P}_{\text {_ }}$ 0.062) may also be relevant, but failed to reach statistical signifi cance (Table 3). In all combinations of two chronic conditions with a statistically signifi cant negative impact on SRH, either severe headache or severe back pain was present .

\section{DISCUSSION}

\section{Main results}

Most women aged 70 - 74 years reported one or more chronic conditions. The more chronic conditions women suff ered from, the lower women rated their health . Although logical and acknowledged (7), this confi rms the essentiality to take multimorbidity into account in the health management of elderly women. We found that all combinations including severe headache and some combinations including severe back pain and another chronic condition had a signifi cantly more negative impact on SRH. This impact was stronger than can be expected from the impact of two or more chronic conditions on SRH .

These conditions are commonly seen in general practice in elderly women, and their presence is largely invisible to the GP due to embarrassment, inconvenience, believing the doctor could not help or acceptation and adaptation by the elderly female patient $(15-17)$. 
Vos, H.M.M., Bor, H.H., Rangelrooij-Minkels, M.J.A., Schellevis, F.G., Lagro-Janssen, A.L.M. Multimorbidity in older women: the negative impact of specific combinations of chronic conditions on self-rated health. European Journal of General Practice: 2013, 19(2), 117-122

\section{Interpretation}

When possible negative eff ects of the most prevalent combinations of chronic conditions on SRH were explored, we found that all signifi cant combinations included severe back pain or severe headache. Findings concerning severe back pain were similar to previous studies that concluded that back pain was negatively associated with SRH $(7,18,19)$. One study concluded that back pain was one of the most important comorbid conditions aff ecting SRH (20), but in another study this negative impact of musculoskeletal systems on SRH was not confi rmed (7).

Previous studies focusing on the eff ect on SRH of comorbidity and headache were not conclusive. Xuan et al., did not fi nd an eff ect on SRH of the combination of a principal disease and migraine (20), whereas Jensen and Stovner stated in their review that the profound co morbid disorders of severely aff ected patients with headache complicate their overall outcome (21). Wiendels et al., found that high headache frequency and comorbidity contribute to a low quality of live in these patients (22). Whether this is a signifi cantly more negative eff ect than expected is unknown, because this eff ect was not compared to other conditions. When studying migraine and co morbidity, Terwindt et al., found that migraineurs are more often depressed than nonmigraineurs, but whether this combination mediated or confounded the SRH ratings of migraineurs went beyond the scope of their study (23) .

Lastly, Lipton et al., also found that depression itself reduces SRH in subjects with migraine, but could not fully disentangle the separate and joint infl uences of migraine and depression (24) .

Studies to determine eff ects of multimorbidity and co morbidity on SRH are not new. Earlier research showed a negative eff ect on quality of life in the combination of chronic respiratory disease, cardiovascular diseases and diabetes $(7,9,10)$. In this study was found that combinations with severe back pain and severe headache, usually considered being less critical, have a signifi cantly negative impact on SRH. Diff erences in methodology are one of the possible reasons why the results diff er from earlier studies. The infl uence of mostly separate chronic condi tions was assessed, whereas others used disease categories $(7,9,10)$ or only physical conditions (10). By combining individual chronic conditions into groups of diseases, the numbers of respondents per group are larger than in this study, which made it harder to fi nd signifi cant correlations. In this study, as individual subgroups of conditions were sometimes small, not all possible combinations of chronic conditions could be studied. Therefore, we limited ourselves to the most prevalent combinations. A second diff erence is that only women were studied, and respondents were older: $59.0,58.5$ and 57.6 years old in previous studies $(7,9,10)$ versus 71.9 years old in this study. We, therefore conclude, that the infl uence of multimorbidity on SRH transforms from combinations of mostly fatal to mostly non-fatal, but disabling diseases as women grow older. Women who have reached the age of $70-74$ have survived several fatal diseases, which usually occur at an earlier age .

Consequently, it is obvious that among women in this age group disabilities related to combinations of chronic non-fatal diseases are more important determinants of SRH than fatal diseases .

Gender eff ects were studied by Rijken et al., (10) as well but they did not study co morbidity with back pain or headache. The other researchers did not study gender, 
Vos, H.M.M., Bor, H.H., Rangelrooij-Minkels, M.J.A., Schellevis, F.G., Lagro-Janssen, A.L.M. Multimorbidity in older women: the negative impact of specific combinations of chronic conditions on self-rated health. European Journal of General Practice: 2013, 19(2), 117-122

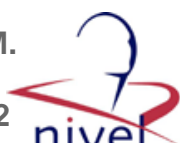

which stresses the importance that correlations can diff er by gender and, therefore, analyses have to be performed separately for men and women .

\section{Strengths and weaknesses of the study}

The strength of this study is the focus on a group of women with good access to GPservices, providing many data on health and health behaviour. By using a nationwide representative survey (the DNSGP-2), a high response was reached. A limitation is that the chronic conditions were self-reported. This implies a GP- confi rmed diagnosis was missed. This may lead to an underestimation or overestimation of the conditions. However, it is questionable whether a non-self-reported chronic condition aff ects SRH. Another limitation is the defi nition of depression and anxiety. In the questionnaire, the question was whether the respondent ever felt very depressed of very anxious for more than two weeks, but other symptoms of a depression or an anxiety disorder were not part of the questionnaire and thus unknown. Nevertheless, Reme and Eriksen found that a single depression-question identifi ed most of the depressive symptoms of a larger rating scale and, therefore, can be considered an indicator of depression (25) .

Lastly, the method of measuring the SRH-ratio used is unorthodox. Even though a high response was reached, the group of women with only one chronic condition was too small to measure a ratio by taking the rate of women with the combination of two chronic conditions with a high SRH, and the mean SRH of women with the two conditions separately. Of the 315 women, only 71 had one chronic condition and the most prevalent single condition without multimorbidity was anxiety in 14 women . By using this method, we realize that we made an underestimation of the impact of multimorbidity on SRH, which only strengthens the conclusions .

Eff ects of chronic conditions on SRH were found. Of course, we have to be very modest when interpreting crosssectional studies and causality. It is diffi cult to say whether causal direction goes from severe headache or severe back pain to low SRH or the other way around. Nevertheless, a signifi cant relation was found between severe headache or severe back pain in combination with a chronic condition and $\mathrm{SRH}$, which needs further attention.

\section{Implications}

Embarrassment, inconvenience, or acceptation and adaptation by elderly women make conditions like headache and back pain largely invisible to the GP $(15,16,17)$. Therefore, an active role of the GP is crucial to determine the extent and severity of both severe headache and severe back pain to act. Future research on the eff ects of treatment of severe back pain and severe headache on the SRH of older women with co morbidity is recommended, preferably by randomized controlled trial.

\section{CONCLUSION}

All combinations including severe headache and some combinations including severe back pain and another chronic condition, have a signifi cantly more negative impact on SRH in women aged $70-74$ years than expected. Taking into account that hidden pathology such as severe headache and severe back pain in combination with a chronic condition is a trigger for low SRH, GPs should be very alert on these hidden conditions in women aged $70-74$ years to add to the quality of the later years of life. 
Vos, H.M.M., Bor, H.H., Rangelrooij-Minkels, M.J.A., Schellevis, F.G., Lagro-Janssen, A.L.M. Multimorbidity in older women: the negative impact of specific combinations of chronic conditions on self-rated health. European Journal of General Practice: 2013, 19(2), 117-122

\section{Declaration of interest:}

The authors report no confl icts of interest. The authors alone are responsible for the content and writing of the paper.

\section{REFERENCES}

1. Van den Akker M, Buntinx F, Roos S, Knottnerus JA . Problems in determining occurrence rates of multimorbidity . J Clin Epidemiol .

$2001 ; 54: 675-9$.

2. Murtagh KN, Hubert HB. Gender diff erences in physical disability among an elderly cohort . Am J Public Health 2004 ; 94 : 1406 - 11 .

3. Ta ş U, Verhagen AP, Bierma-Zeinstra SMA, Hofman A, Odding E, Pols HAP, et al . Incidence and risk factors of disability in the elderly: The Rotterdam Study . Prev Med . $2007 ; 44: 272-8$.

4. Oman $D$, Reed $D$, Ferrara A. Do elderly women have more physical disability than men do? Am J Epidemiol. 1999; 150: 834- 42 .

5. I dler E L, B enyamini Y. S elf-rated health and mortality: A review of twenty-seven community studies. J Health Soc Behav . $1997 ; 38: 21-37$.

6. DeSalvo KB, Bloser N, Reynolds K, He J , Muntner P. Mortality prediction with a single general self-rated health question .

A meta-analysis. J Gen Intern Med. 2006; 21: 267- 75 .

7. Fortin M, Dubois MF, Hudon C, Soubhi $\mathrm{H}$, Almirall J . Multimorbidity and quality of life: A closer look. Health Qual Life Outcomes $2007 ; 5: 52$.

8. G ijsen R, H oeymans N, S chellevis F G, R uwaard D, S atariano W A, van den Bos GAM . Causes and consequences of comorbidity: a review . J Clin Epidemiol. 2001; 54: 661- 74 .

9. R ijken M , v an Kerkhof M, D ekker J , S chellevis F G. C omorbidity of chronic diseases: eff ects of disease pairs on physical and mental functioning . Qual Life Res . $2005 ; 14$ : 45 -55 .

10. Bayliss EA, Bayliss MS, Ware JE, Steiner JF. Predicting declines in physical function in persons with multiple chronic medical conditions: What we can learn from the medical problem list .

Health Qual Life Outcomes $2004 ; 2: 47$.

Fortin M, Bravo 11. G, Hudon C, Vanasse A, Lapointe L. Prevalence of multimorbidity among adults seen in family practice. Ann Fam Med . $2005 ; 3: 223-8$.

12. Westert GP, Schellevis FG, de Bakker DH , Groenewegen PP, Bensing JM, van der Zee $\mathrm{J}$. Monitoring health inequalities through general practice: The Second Dutch National Survey of General Practice. Eur J Public Health 2005; 15: 59- 65.

13. Ware JE, Sherbourne CD . The MOS 36-item short-form health survey (SF-36). I. Conceptual framework and item selection. Med Care $1992 ; 30: 473-83$.

14. A aronson N K, M uller M , C ohen P D, E ssink-Bot M L, F ekkes M, Sanderman R, et al . Translation, validation, and norming of the Dutch language version of the SF-36 Health Survey in community and chronic disease populations. J Clin Epidemiol. 1 998; 5 1: 1 05568 .

15. C ampbell C , C ramb G. ' N obody likes a back bore' - e xploring lay perspectives of chronic pain: Revealing the hidden voices of nonservice users. Scand J Caring Sci . 2008 ; $22: 383-90$.

16. Picavet HSJ , Schouten JSAG . Musculosceletal pain in the Netherlands: Prevalences, consequences and risk groups, the DMC(3)-study .

Pain 2003; 102: 167- 78 .

17. Thomas E , Boardman HF , Ogden H, Millson DS , Croft PR . Advice and care for headaches: Who seeks it, who gives it? Cephalalgia $2004 ; 24: 740-52$.

18. Sirola J , Tuppurainen M , Rikkonen T, Honkanen R, Koivumaa- Honkanen H, Kr oger $\mathrm{H}$. Correlates and predictors of self-rated health and ambulatory status among elderly women - Cross-sectional and 10 years population-based cohort study . Maturitas 2010; 65: 244- 52 . 
Vos, H.M.M., Bor, H.H., Rangelrooij-Minkels, M.J.A., Schellevis, F.G., Lagro-Janssen, A.L.M. Multimorbidity in older women: the negative impact of specific combinations of chronic

19. Hartvigsen J , Christensen K, Frederiksen H. Back and neck pain exhibit many common features in old age: a population-based study of 4,486 Danish twins 70-102 years of age . Spine $2004 ; 29: 576-80$.

20. Xuan J , Kirchdoerfer LJ , Boyer JG , Norwood GJ . Eff ects of comorbidity on healthrelated quality-of-life scores: an analysis of clinical trial data . Clin Ther. 1999; 21: 383- 403

21. J ensen R , S tovner L J. E pidemiology and comorbidity of headache .

Lancet Neurol. 2008; 7: 354-61.

22. Wiendels NJ , van Haestregt A, Knuistingh Neven A, Spinhoven P, Zitman FG , Assendelft WJJ, et al. Chronic frequent headache in the general population: Comorbidity and quality of life . Cephalalgia 2006; 26: 1443- 50 .

23. T erwindt $G M, F$ errari $M D, T$ ijhuis $M, G$ roenen $S M A, P$ icavet $H S J$, Launer LJ. The impact of migraine on quality of life in the general population. The GEM study . Neurology $2000 ; 55: 624-9$.

24. Lipton RB , Hamelsky SW , Kolodner KB, Steiner TJ , Steward WF .

Migraine, quality of life, and depression. A population-based case-control study . Neurology $2000 ; 55: 629-35$.

25. Reme SE , Eriksen HR . Is one question enough to screen for depression? Scand J

Public Health 2010 ; 38 : $618-24$. 